\section{Chemistry at the University of Edinburgh}

The Playfair Collection and the Teaching of Chemistry at the University of Edinburgh, 1713-1858. By R. G. W. Anderson. Pp.175. (The Royal Scottish Museum: Edinburgh, 1978.). Paperback $£ 4.50$.

SHORTLY after Lyon Playfair's appointment in 1858 to the Edinburgh Chair of Chemistry he presented to what is now the Royal Scottish Museum 75 diverse pieces of scientific apparatus from the University Chemical Laboratory which he believed to be of historical significance. Sixty-four of these items, thought to be associated with Black, Hope and Gregory, are extant and comprise the Playfair Collection. It is an important collection, as few pre-nineteenth century chemical artefacts of known provenance survive.

Dr Anderson devotes about half of his book to dicussing the provenance of the Playfair Collection as a whole and to describing fully each item, the first detailed discussion of its kind to be published. To provide a context for this catalogue the first half of the book describes the teaching of chemistry at Edinburgh from 1713 to 1858; during most of this period the University of Edinburgh was pre-eminent in Britain in the teaching of Chemistry, thus making Anderson's book of interest to both students of early scientific apparatus and historians of science.

In the catalogue section, the entry for each item consists of physical description, illustration, brief history of the development of the class of apparatus and, for some items, evidence for associating it with a particular Professor. The illustrations, both photographs and diagrams, are excellent and the brief histories are scholarly and wellreferenced. The very difficult task of substantiating the association of a particular item with a particular Professor is capably done but, as is probably inevitable, the evidence is sometimes less than convincing.

The first half of the book, the author states, is not intended to be a history of the teaching of chemistry at Edinburgh or of those who taught it. This section deals primarily with their laboratories, their acquisition of apparatus and, to a certain extent, what and how they taught. In his principal aims the author succeeds admirably while supplying sufficient biographical material to make a continuous and readable account; it is particularly valuable to have hitherto unpublished information on the first holder of the Chair, Crawford, and extensive new information on his successor, Plummer, both of whom, other than in Underwood's Boerhaave's Men, have received little scholarly attention in contrast to their successors, Cullen, Black, Hope and Gregory. Anderson's account is well-referenced, mainly to primary sources, and is very lucid. The identified with very extensive evidence. Perhaps of wider general interest are the types of apparatus acquired, the costs thereof and the sources of supply; here again the author is to be congratulated on the range and depth of his research. Less well done is the account of the teaching which, though adequate as to the method of teaching, gives little information on the content of the courses. There are a few minor errors: for example, Crawford was allotted money not "to put up his stalls" (p4) but his "stells", Old Scots for "stills"; again, Lyon Playfair was a locations of the various laboratories are

candidate for the Edinburgh chair in 1844 (p55) as the Town Council Minutes relating to that appointment show.

One matter of opinion on which I must. express disagreement with the author is his view that Opera Chemica is a student notebook of Plummer's lectures. I believe it to be a set of instructions for the pharmaceutical preparations carried out in Plummer's laboratory; it is difficult to believe from Plummer's two published chemical papers that his chemical lectures. were so narrow as to consist merely of pharmaceutical processes. These minor criticisms do not detract from my view that this is a splendid book which will interest not only the specialist but also the general reader and which at $£ 4.50$ is remarkable value.

W. P. Doyle

W. P. Doyle is Senior Lecturer in Chemistry at the University of Edinburgh, UK.

\section{Origins uncertain}

The Origin of the Solar System. Edited by S. F. Dermott. Pp. 668. (Wiley: Chichester, UK, and New York, 1978.) $£ 28.50$.

WE know and understand less about the Earth's interior than the Sun's. We know less about the origin of the planets than we do about the formation of stars and even (one may feel) of the Universe. This will be apparent to anyone who reads The Origin of the Solar System, the proceedings of a NATO Advanced Study Institute held at the University of Newcastle in 1976, and capably edited by S. F. Dermott.

The reason for this state of affairs is clear enough: we have only one example of a planetary system with closely measured physical properties, whereas we can observe a large number of stellar systems (and it is difficult to falsify statistically predictions about the Universe). Nevertheless, it leaves one uneasy to see a field of science so dominated by speculation, with so little hard observational foundation. For that reason one cannot escape a sense of unreality when reading this volume, which could not have been readily remedied in 1976. The discovery since then of strong candidates for pre-planetary discs around a handful of nearby stars by the Steward Observatory's infrared observing group gives some grounds for hoping that we may be able eventually to test hypotheses against planetary systems observed now to be in the process of forming. Certainly we are learning much about the Sun's birth processes - a subject not given much prominence in the present book - from recent observations of currently forming stars.
Within the book one does find various contributions which are well written and which may serve as excellent introductory reviews of specialised topics. The article by Kirsten on the use of radioactive decay for chronology using samples from the Earth, the moon and meteorites starts with an admirable discussion of principles and their application, and contains exact descriptions of a variety of experiments. One does not need to agree with the minutae in his final cosmochronology to be impressed with the precision, and the well understood limits to that precision, of estimates such as 4.5 to $4.6 \times 10^{9} \mathrm{yr}$ for the epoch of Solar System formation. A complementary paper by Larimer on meteorites in an essentially similar style is also thorough and readable. In a very different type of article Stevenson sets out clearly how global physical observations of the outer planets - their mean densities, gravitational moments and their recently measured internally generated luminosities - set constraints on their physical and chemical structures. One is then left with the problem of how the resulting abundance ratios of hydrogen to helium and to heavier elements came to deviate from their cosmic and especially their solar values. Neglecting such cosmogonic considerations we have models for the interior of Jupiter more reliable and more able to describe observable phenomena (principally its huge magnetosphere) than comparable models for the Earth.

It is on the theoretical side where credibility wears thin. Reeves acknowledges this in his introduction, where he outlines four types of theories for planetary formation, two of which were postulated in their original forms in the eighteenth century, but all of which have their contemporary protagonists. 